\title{
Unmanned Free Balloon Flight's Integration into Unmanned Aircraft System Traffic Management
}

\author{
N. Hegyi ${ }^{1}$, J. Jósvai ${ }^{2}$ \\ Széchenyi István University, Department of Vehicle Manufacturing, \\ Egyetem tér 1, H-9026 Győr, Hungary \\ e-mail ${ }^{1}$ : hegyi.norbert@sze.hu \\ e-mail²: josvai @sze.hu
}

Abstract: This paper describes and inspects unmanned aerial vehicle, unmanned aircraft system, unmanned aircraft system traffic management, light and medium unmanned free balloons. It presents future safety developments and goals of the European Union's own airspace, but also the Hungarian free route airspace. The development and realization of Swiss U-Space is discussed. Hungarian flights statistics are analyzed. Based on statistics, realized and under development solutions there are recommendations in the paper for the integration of the flight tacking for the mentioned balloons. A real flight tracking test has been done and it is shown in this paper. Its properties are analyzed with conclusions for possible usage.

Keywords: unmanned aerial vehicle; unmanned aircraft system traffic management; unmanned free balloon; U-Space; tracking

\section{Introduction}

This paper investigates the possibilities of the integration of light and medium unmanned free balloon flight tracking into unmanned aircraft system traffic management. Firstly, in this introduction the main terms as unmanned aerial vehicle (UAV), unmanned aircraft system (UAS), unmanned aircraft system traffic management (UTM), unmanned free balloons need to be clarified for further understanding. Safety developments for the European airspace are described. The Hungarian free route airspace and Hungarian flights statistics are analyzed. Later on 
a real flight tracking test is shown and its properties (accuracy, continuity, reliability) are analyzed for possible usage. Conclusions are drawn from these results.

\subsection{Unmanned aerial vehicle}

Unmanned aerial vehicle as they name suggests are aerial vehicles, which have no human operating personal on board, and are flying remotely or autonomously. They can be classified by a wide range of features, for example by weight, or engine type. The mass of them ranges from a few grams to multi-ton aircraft [1]. Their flight altitude extends from the ground up to the jet aircrafts'. Rotorcraft designed UAVs are able to float on the spot while the fastest ones can fly with hypersonic speeds [2], [3]. As it can be seen, unmanned aerial vehicles exist in many variants. In this paper the smaller UAVs, commonly called in the last years 'drones' are inspected.

\subsection{Unmanned aircraft system}

An unmanned aircraft system or UAS includes the unmanned aerial vehicles, their control and support systems. Many of them are currently under development [4].

\subsection{Unmanned aircraft system traffic management}

Unmanned aircraft system traffic management or UTM is a management system for the autonomous controlled operations of unmanned aerial systems. The UTMs are now mostly under development [5].

\subsection{Unmanned free balloons}

Unmanned free balloons, as their names suggests are unmanned and fly freely. These devices generally consist three main parts, a balloon, a parachute and a probe. This can be seen in Fig. 1. The photo was taken by N. Hegyi in 2017 at a test flight to check possible technical solution for further flights. 


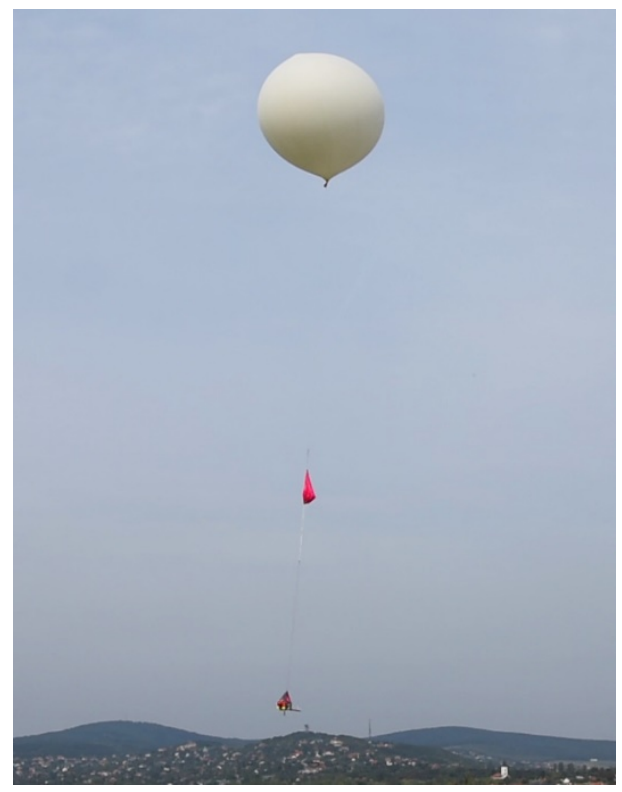

Figure 1. A light unmanned free balloon

Unmanned free balloons should be used in such a way that they do not endanger persons, property or other aircraft. In order to avoid accidents, the balloons need to be designed with a not hazardous structure.

The basic classifying characteristics for light and medium free balloons are:

- They carry a payload of one or more packages with a combined mass of less than $4 \mathrm{~kg}$ (light) or more than $4 \mathrm{~kg}$, but less than $6 \mathrm{~kg}$ (medium);

- $\quad$ they do not contain a package of $3 \mathrm{~kg}$ or more;

- they do not contain a package with a $2 \mathrm{~kg}$ or more with an area density greater than 13 g per square centimeter;

- $\quad$ and the suspension of the load is provided by a rope or other device with requiring an impact force of less than $230 \mathrm{~N}$ to separate the payload from the balloon. [6] 


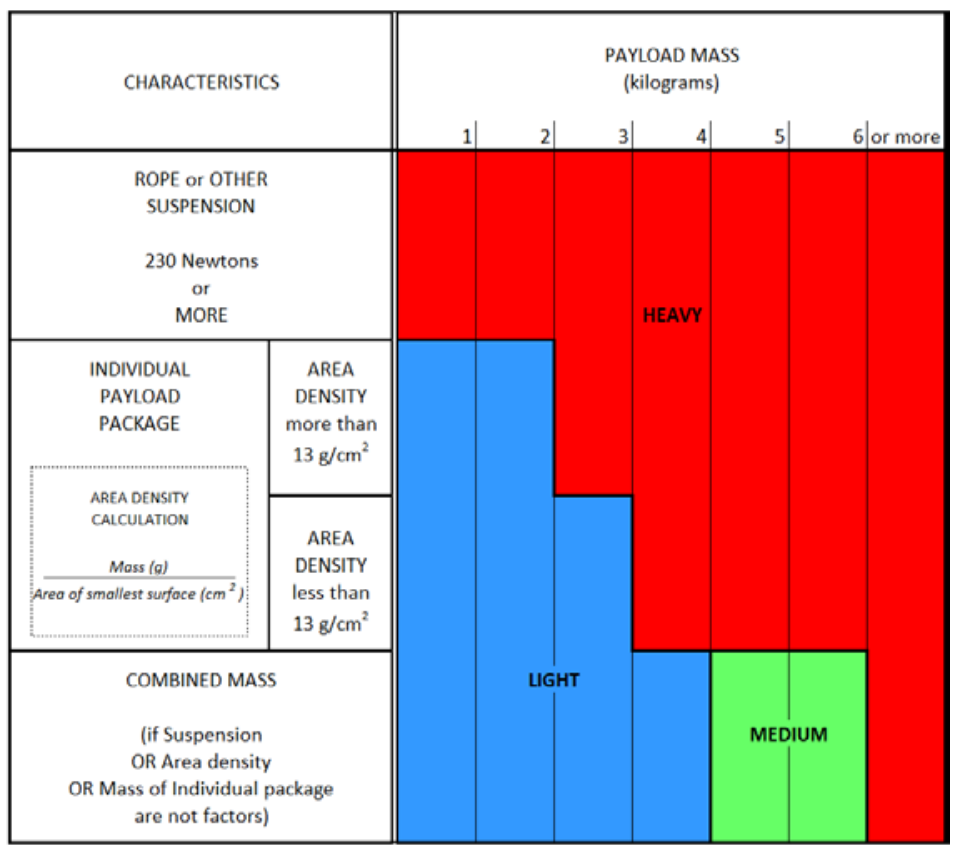

Figure 2. Categorization of unmanned free balloons [6]

This research is concentrating on payloads above $1 \mathrm{~kg}$. In comparison meteorological balloons' radiosondes (which are the payloads) are usually about 40$300 \mathrm{~g}$. To be clear, radiosondes for special purposes can be heavier [7], [8], [9].

\section{Tracking}

\subsection{U-space}

The primary purpose of this paper is to demonstrate the integration of authentic tracking of unmanned light and medium free balloons into UTM. U-space is a concept published in 2017 by Single European Sky Air Traffic Management Research (short form: SESAR), which aims the use of UTM through Europe [10].

A previous work by N. Hegyi [11], had already described tracking systems out of these several were used for paragliding, some for drones and some for professional airplane flights. It concluded that the technology used by smartphones could be used partially for the tracking of light and medium free balloons.

In September 2017, the first U-space system was realized in Switzerland. This proved that air navigation services will be capable for the integration of the UAS 
into their systems. The goal is to create a system for safe, efficient, and secure access to the airspace for the drones. In a short way the goal is to increase the safety of airspace [12]. This demonstration showed for example in practice the use of eregistration and e-identification, airspace authorization, flight planning, flight management, live telemetry and tracking, the integration into the Swiss Air Traffic Service (ATS) radar system [13].

In March 2018 it was announced that Skyguide (the Swiss air traffic service provider) and AirMap (an airspace management platform for UAVs) will develop and deploy the first national drone traffic management system in Europe [14].

\subsection{Free route airspace}

Euro-control initiated the development of free route airspace in 2008. The free route airspace (FRA) is a specified airspace. In this airspace a flight route can be planned and defined via an entry point and an exit point, with possible intermediate waypoints for routing. This is possible without any references to the air traffic services route network. In this kind of airspace, the flights are still the subject to air traffic control [15].

In the Commission Implementing Regulation No. 716/2014 the European Union gave a deadline for January 1st 2022 to introduce the FRA [16]. On February 5th 2015, HungaroControl was the first one in Europe to introduce the FRA under the name Hungarian Free Route Airspace or HUFRA [17].

\subsection{Air traffic data}

The need for integrating of light and medium unmanned free balloons to UTM is supported by flight data.

From Table I in the 'all flights' column (it contains all flights above, to and from Hungary) increased by $31.25 \%$ in comparison of August 2015 to August 2018 [18], [19], [20].

Table 1. Number of flights for statistics

\begin{tabular}{|c|c|c|c|}
\hline & $\begin{array}{c}\text { Flights over } \\
\text { Hungarian } \\
\text { airspace }\end{array}$ & Budapest flights & All flights \\
\hline August 2015 & 74201 & 8685 & 82886 \\
\hline August 2016 & 76237 & 8988 & 85225 \\
\hline August 2017 & 81613 & 9681 & 98133 \\
\hline August 2018 & 90198 & 11049 & 108791 \\
\hline
\end{tabular}


As it can be seen in Fig. 3 the yearly overflying traffic above Hungary increased by $71.56 \%$ from 2004 to 2017 [21].

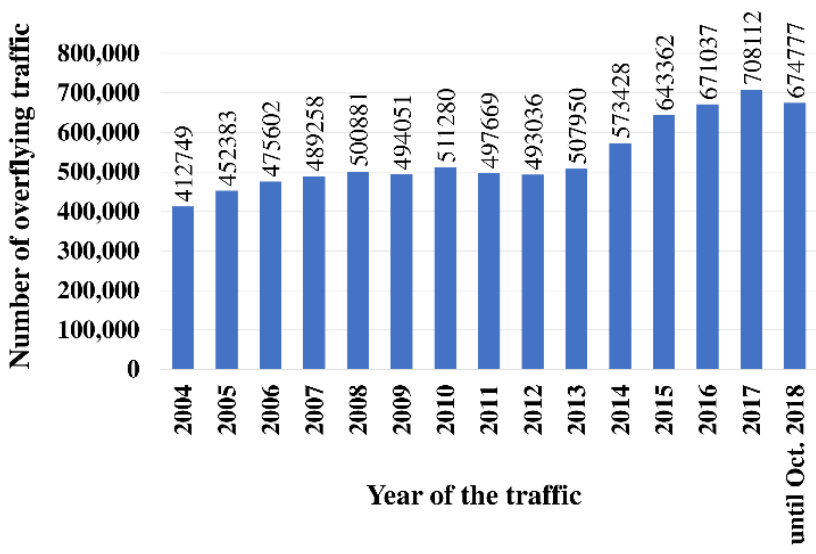

Figure 3. Overflying traffic data above Hungary [21]

As it could be seen in Table I and Fig. 3 the number of flights is increasing, which means that the flight density in airspace is also increasing. In addition, the introduction of HUFRA caused the elimination of specified ATS routes in those defined airspaces. In a sense this causes the possibility to get anywhere in the free route airspace near an airplane. The bigger flight density and the FRA influence in indirect way to increase the danger of a collision by a light or a medium unmanned free balloon with an aircraft.

\subsection{Dangerous proximity}

In the previous mentioned study by N. Hegyi [11] a near collision of a hobby light unmanned free balloon and an airplane above the USA was mentioned. The balloon got 152 meters close (500 feet) under the jet. This can be seen for far enough, but if an ascent rate of $5 \mathrm{~m} / \mathrm{s}$ is assumed for a similar situation, and 30 seconds later for the aircraft's arrival at that point than it could have been much closer.

Light unmanned free balloons are not seen on radar by HungaroControl civil air traffic controllers. They are flying without any direct guidance, so only a controlled drone or a jet aircraft can avoid any hazardous close ups or collisions. This paper recommends that if the balloons mentioned here would also contain the electronics required for U-Space, they would increase safety in the airspace, beginning from their release until their landing, in order to avoid potential collisions. 
The problem currently is, that the initial phase of U-space systems is working just to a few hundred meters in altitude [10]. The next practical step in this research is to develop a solution for UTM up to an altitude of $18 \mathrm{~km}$. The goal of this altitude is justified by next generation supersonic business aircraft currently in concept studies or under development. Their preferred flight altitude will be $17 \mathrm{~km}$ [22].

\section{A concept}

Previously dangers caused by unmanned free balloons to manned airplanes, like dangerous proximity or the possibility of collisions were already mentioned in this study. The primary objective would be to track the balloons and integrate their positions and flight routes into the UTM.

In the present, for safe flights at the near ground phase of their flights a proper tracking is possible with mobile phone-based technology. This solution would work up to the maximal operating height above ground of 3G-4G mobile phone networks and the equipment used for the 'first steps of U-Space'.

There is also the flight phase of higher altitudes, like $10 \mathrm{~km}, 20 \mathrm{~km}$ above ground or even more. Above the altitude of currently developed U-space there are also some possible solutions. Currently this paper would recommend the use of other radio communication systems.

For practical testing Automatic Packet Reporting System (APRS) amateur radio frequencies are usable. In Hungary one of these frequencies is $144.8 \mathrm{MHz}$. It needs to be mentioned that APRS tracking is accessible in real-time with Automatic Packet Reporting System-Internet Service (APRS-IS) for example on the website 'aprs.fi'. For realistic testing the HABP42+ balloon tracking device was used for active paragliding flights on 19th and 20st April 2019 near Tolmin in Slovenia. The call sign was N. Hegyi's HA1NX. The flight of 20th April 2019 is a positive demonstration for the APRS tracking. The device was set to transmit tracking data every 70 seconds with a delay of a few seconds (caused intentionally by a few settings). The analyzed data show that after the take off the time between transmissions was 76-80 seconds. Four times this was longer, caused by no possible contact to internet connected receiver stations. The mentioned situations will be analyzed later in this document.

On Fig. 4. the test flight of the 20th April 2019 is shown on GoogleEarth map with satellite image layer. The blue line is the flight route shown by APRS tracking data. The red colored path is the real flight route recorded by the device on a memory card. The flight lasted for 1 hour 9 minutes, its length was about $22.9 \mathrm{~km}$ measured in 3 dimensional space. The flight started from a $1085 \mathrm{~m}$ high mountain, the maximum altitude was $2059 \mathrm{~m}$, the landing point was at $225 \mathrm{~m}$ in a valley. The average speed was $26.05 \mathrm{~km} / \mathrm{h}$, the maximum was $56.21 \mathrm{~km} / \mathrm{h}$. 


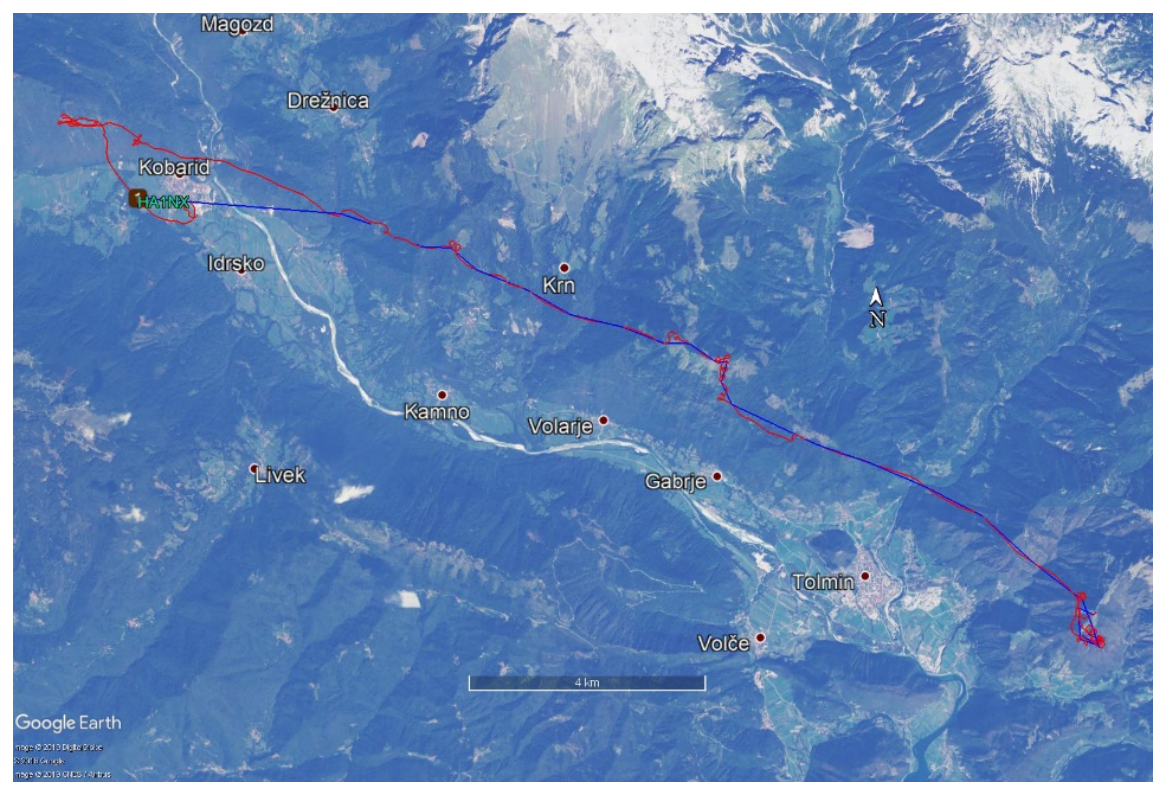

Figure 4. Flight tracking test shown

The beginning phase of the flight is shown on Fig. 5 . The blue line is the flight route shown by APRS tracking. The red colored sign - the elevating spiral connected with vertical lines to the ground - is the real flight route recorded by the device on a memory card. The first 12 minutes of the flight can be seen on the image. The spiral flight route is caused by the special paragliding elevation technique with the upward moving air. A spatial difference between the blue and red lines can be seen. The APRS tracking sent a data package every 76-80 seconds and between these packages the real movement was not a straight line, it took place on curved lines. This difference is between 0 to 200 meters. In case of a light or a medium unmanned balloon's flight this difference would be most probably under the 200 meters value. This would be the result, because a balloon's flight has no spiral movement compared to paragliding. 
N. Hegyi and J. Jósvai - Acta Technica Jaurinensis, Vol. 12, No. 4, pp. 321-334, 2019

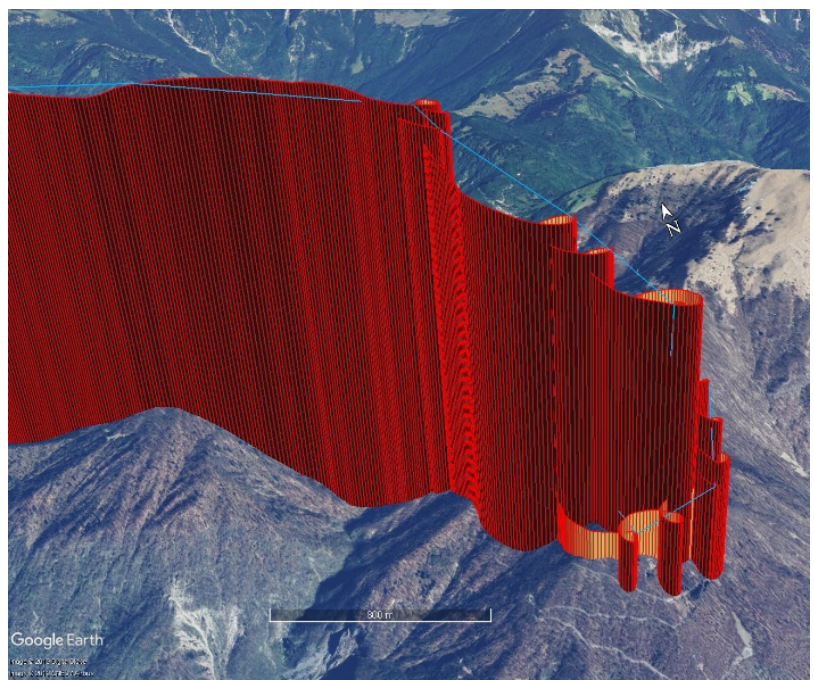

Figure 5. The beginning phase of the flight

On Fig. 6. the middle parts of the flight can be seen. This image shows the difference of the APRS tracking and the real flight in the middle parts of the flight. The red spiral flight route is not connected to the ground.

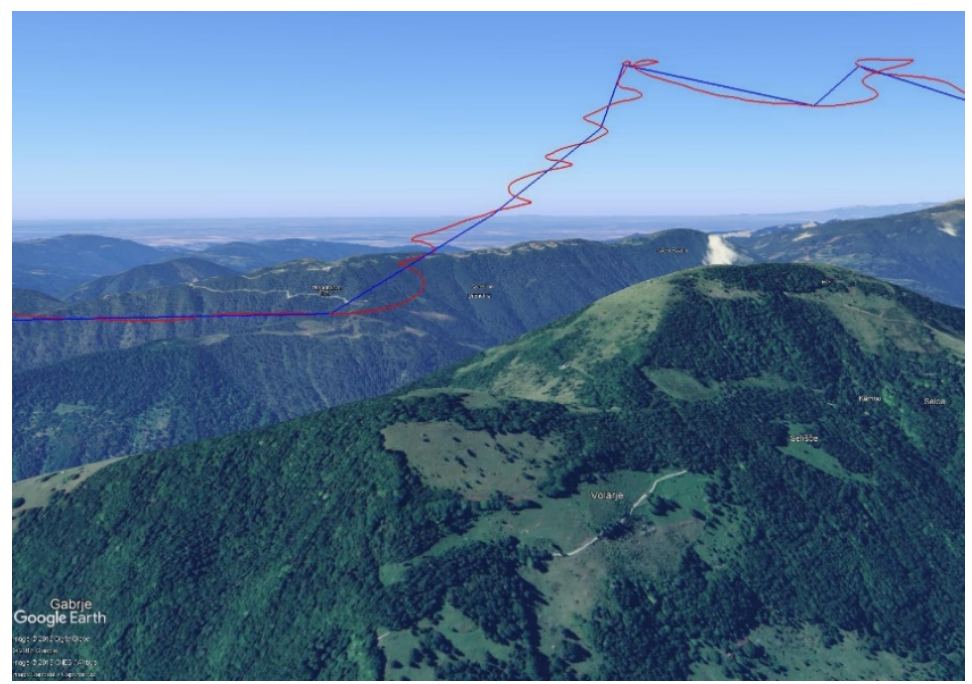

Figure 6. The beginning phase of the flight 
On Fig. 4., Fig. 5. and Fig. 6. the difference between the transmitted tracking route and the real flight route can be seen. From the analyzed data it can be seen, that the difference was mainly $0-200 \mathrm{~m}$, with $2 \mathrm{~km}$ partly at the last 17 minutes.

As it was mentioned previously, that the connection to the APRS was lost four times. Two times the time between two tracking points were 2 minutes 40 seconds. This means that, the connection was lost for one tracking data package. It follows that one tracking data package has been sent by the tracking device, but there was no receiver station in connection range for getting the data. The difference between the reported and real flight route was $300-350 \mathrm{~m}$ which is not significantly bigger than on the more accurate tracking parts.

On Fig.7. the end part of the flight route is shown at the 16 minutes without contact is shown. At that moments on GoogleEarth it can be seen, that mountains were between the paraglider and all receiver stations. The biggest difference between real and received tracking is $2 \mathrm{~km}$. Despite of low probability this inaccuracy could cause dangerous proximity between balloons and other aircrafts. This could be solved with a more dense planned network or with a two-mode solution described after Fig. 8.

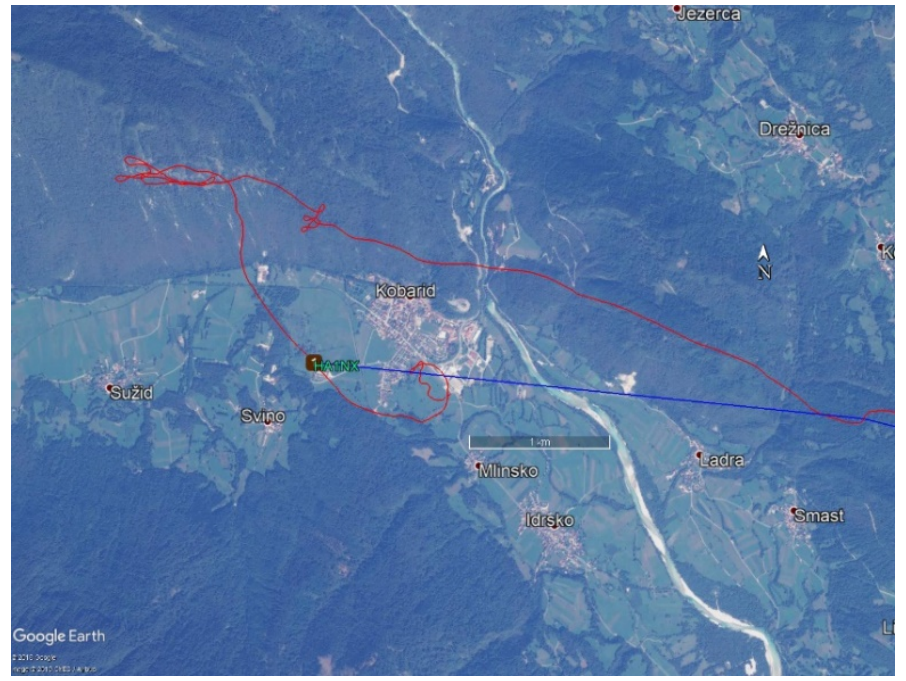

Figure 7. The end part of the flight

On Fig. 8. the same part of the flight is shown on a topographic map, on which the mentioned internet connected receiver stations are also shown [23]. On this map the causes of contact lost can be seen, which are the topographic conditions. At point "A" the connection was made with station S56VIL-10, after this it was out of range caused by the terrain. At point "B" the link was made with station S55AOP-5 (outside the map) from 1094m altitude. After point "B" the tracking connection was 
missing for 16 minutes 56 seconds. At point "C" the connection was made again with S55AOP-5 from $631 \mathrm{~m}$ altitude. After point "C" the tracking was missing at the last 5 minutes. The difference between APRS tracking and the real route was at maximum $2 \mathrm{~km}$ at "B-C" flight and $1 \mathrm{~km}$ after "C".

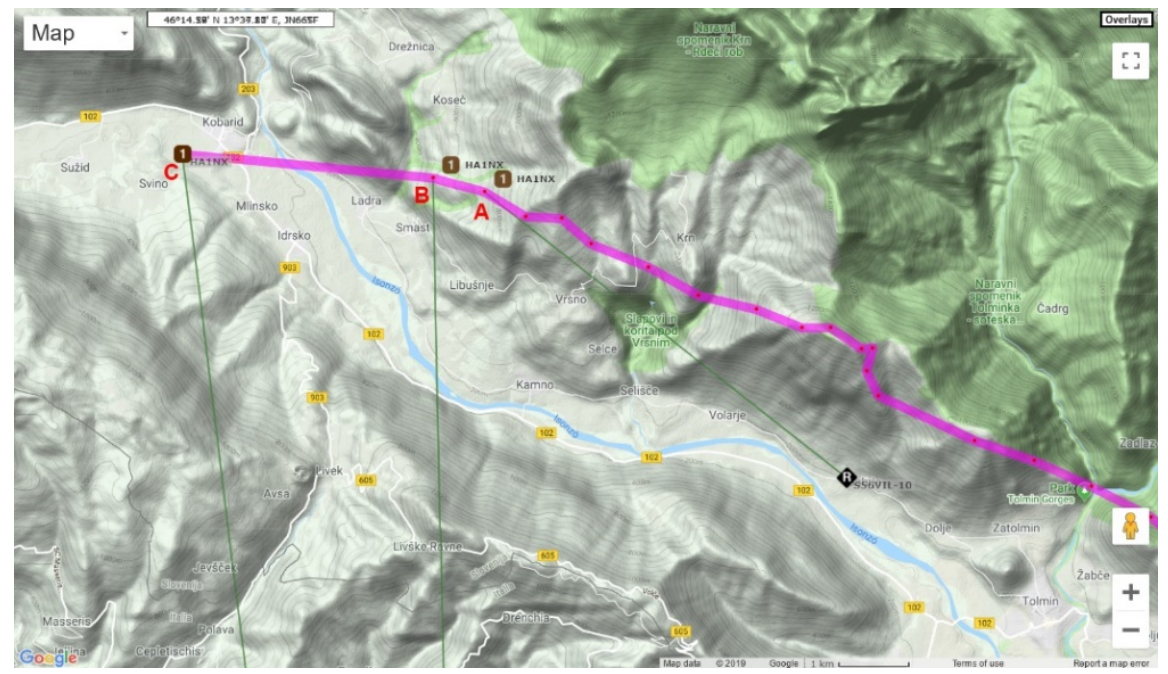

Figure 8. The end part of the flight without connection

With a two-mode tracking system the tracking would be possible by APRS systems at high altitudes, and by 3G-4G mobile systems near ground. The tracking data could be sent by mobile phone-based equipment from altitudes like the APRS untracked last 5 minutes of the flight. To prevent the lose of APRS-IS contact, the improvement of the APRS receiver stations would be needed. On first order, this means more stations, on calculated, planned positions. If this development would be realized, then this technology would be a good option for further usage.

There is a problem with one part of this kind of tracking that needs to be mentioned. A call sign alone does not make the tracking authentic or from another perspective it does not make it enough official for air traffic controllers. There are laws for amateur radio communication which forbid improper use of call signs, but this does not mean, that this would not be possible. It is necessary to search for more authentic solutions [24].

In order to achieve the integration of light and medium unmanned free balloons into UTM, in addition to the necessary tracking equipment an official registration would be needed. This would make the identification of the balloon and the operator possible. 


\section{Conclusion}

At the end of this study the conclusion can be that a two-mode (near ground and high-altitude mode) tracking system should be developed. By the test presented and analyzed in this study it is confirmed, that it can be achieved with further development of already existing tools adapted for this kind of special usage. Near ground mode would be working with 3G-4G mobile telephone technology, and the high-altitude mode would use APRS radio communication. The big step to assure a system, which can be used officially in an authentic way to integrate it into UTM is necessary.

\section{Acknowledgement}

The paper was written with the support of the project titled 'Internationalization, initiatives to establish a new source of researchers and graduates and development of knowledge and technological transfer as instruments of intelligent specializations at Széchenyi István University (project number: EFOP-3.6.1-16-2016-00017).

\section{References}

[1] M. Hassanalian, A. Abdelkefi, Classifications, applications, and design challenges of drones: A review, Progress in Aerospace Sciences, 91 (2017) pp. 99-131.

doi: https://doi.org/10.1016/j.paerosci.2017.04.003

[2] Airbus, Zephyr (2018), [cited 2018-11-30]

URL https://www.airbus.com/content/dam/corporatetopics/publications/brochures/0296_18_2_ZEPHYR_datasheet_E_horizontal A4.pdf

[3] NASA, X-43A Hyper-X (2018), [cited 2018-11-30] URL https://www.nasa.gov/centers/armstrong/history/experimental_aircraft/X43A.html

[4] S. G. Gupta, M. M. Ghonge, P. M. Jawandhiya, Review of unmanned aircraft system (UAS), International Journal of Advanced Research in Computer Engineering \& Technology, 2 (4) (2013) pp. 1646-1458.

[5] T. Jiang, J. Geller, D. Ni, J. Collura, Unmanned aircraft system traffic management: Concept of operation and system architecture, International Journal of Transportation Science and Technology, 5 (3) (2016) pp. 123-135. doi: https://doi.org/10.1016/j.ijtst.2017.01.004 
[6] EUR-Lex, Commission implementing regulation (EU) No 923/2012, [cited 2018-12-01]

URL https://eur-lex.europa.eu/legalcontent/EN/TXT/PDF/?uri=CELEX:32012R0923\&from=EN

[7] Vaisala, Radiosonde RS41-D Datasheet B211609EN-D (2018), [cited 201812-08]

URL https://www.vaisala.com/sites/default/files/documents/RS41-DDatasheet-B211609EN.pdf

[8] Meisei Electric. iMS-100 GPS Radiosonde, meteorology, Products (2018), [cited 2018-11-30]

URL http://www.meisei.co.jp/english/products/ims-100-e.pdf

[9] Intermetsystems, iMet-1-ABxn Radiosonde (2018), [cited 2018-11-30] URL http://www.intermetsystems.com/ee/pdf/202060_iMet-1ABxn_Data_161006.pdf

[10] SESAR, European drones outlook study - Unlocking the value for Europe, 2016, [cited 2018-02-28]

URL

https://www.sesarju.eu/sites/default/files/documents/reports/European_Drone s_Outlook_Study 2016.pdf

[11] N. Hegyi, Safety issues for light and medium unmanned free balloons tracking, Repüléstudományi Közlemények, 30 (2) (2018) pp. 217-224, in Hungarian.

[12] Skyguide, Skyguide demonstrates integration of drones into airspace by means of the U-space (2017), [cited 2018-09-10]

URL https://www.skyguide.ch/en/events-media-board/news/\#p9260-9267$\underline{9277}$

[13] Airmap, In Switzerland, U-space takes off (2017), [cited 2018-09-10]

URL https://www.airmap.com/switzerland-u-space-skyguide-demo/

[14] Skyguide, Skyguide \& AirMap join forces to develop Europe's first national drone traffic management system (2018), [cited 2018-09-10]

URL https://www.skyguide.ch/en/events-media-board/news/\#p13446-13453$\underline{13459}$ 
[15] Eurocontrol, Supporting European Aviation (2018), [cited 2018-11-30] URL https://www.eurocontrol.int/articles/free-route-airspace

[16] EUR-Lex, Commission Implementing Regulation (EU) No 716/2014, [cited 2018-10-20]

URL https://eur-lex.europa.eu/legalcontent/EN/TXT/?uri=uriserv\%3AOJ.L_.2014.190.01.0019.01.ENG

[17] HungaroControl, The most effective version of free airspace management is introduced by HungaroControl firstly in Europe (in Hungarian) [cited 201810-20]

URL https://www.hungarocontrol.hu/sajtoszoba/hirek/hungarian-free-routeairspace

[18] Hungarian Air Traffic Statistics, HC Radar - HungaroControl’s Magazine, September 2016, p. 17.

[19] Hungarian Air Traffic Statistics, HC Radar - HungaroControl’s Magazine, September 2017, p. 17.

[20] Hungarian Air Traffic Statistics, HC Radar - HungaroControl’s Magazine, September 2018, p. 17.

[21] HungaroControl's Flight Statistics [cited 2018-10-20]

URL https://en.hungarocontrol.hu/about-us/air-navigation-services/statistics

[22] Y. Sun, H. Smith, Review and prospect of supersonic business jet design, Progress in Aerospace Sciences, 90 (2017), pp. 12-38.

doi: http://dx.doi.org/10.1016/j.paerosci.2016.12.003

[23] aprs.fi, [cited 2019-04-25]

URL

https://aprs.fi/\#!mt=roadmap\&z=13\&ts $=1555718400 \&$ te $=1555804800 \&$ call $=$ a\%2FHA1NX

[24] 15/2013 (IX. 25.) NMHH Decree on Amateur Radio Service (in Hungarian) [cited 2018-12-19]

URL https://net.jogtar.hu/printiframe?docid=A1300015.NMH\&targetdate=\&printT itle=15/2013.\%20\%28IX.\%2025.\%29\%20NMHH\%20rendelet 